\title{
Studies on Barlow points, Gauss points and Superconvergent points in 1D with Lagrangian and Hermitian finite element basis
}

\author{
DAVID SOARES PINTO JÚNIOR \\ Department of Mathematics, Federal University of Sergipe \\ 49100-000 São Cristóvão, SE, Brazil \\ E-mail: david@ufs.br
}

\begin{abstract}
In this work, we consider the superconvergence property of the finite element derivative for Lagrange's and Hermite's Family elements in the one dimensional interpolation problem. We also compare the Barlow points, Gauss points and Superconvergence points in the sense of Taylor's Series, confirming that they are not the same as believed before. We prove a not evident and new superconvergence property of Hermite's basis as well which shows that the centroid is not only a superconvergent for $u_{h}^{\prime}$ but an $O\left(h^{5}\right)$ accuracy point.
\end{abstract}

Mathematical subject classification: $74 \mathrm{~S} 05$.

Key words: Gauss points, Barlow points, Superconvergence points, derivative, finite elements.

\section{Introduction}

A mathematical argument to prove the existence of points where a higher order accuracy for derivatives exists is implied from the Rolle's theorem or the Mean Value Theorem for derivatives. Let $u \in C^{0}(\widehat{I})$ and $u_{h}$ be a lagrangian finite element interpolant of $u \in \widehat{I}$. Then, $u_{h}\left(x_{i}\right)=u_{i}=u\left(x_{i}\right), i=0, \ldots, k$ and internally the field $u$ is interpolated as $u_{h}(x)=\sum_{i=0}^{k} L_{i}(x) \cdot u_{i}$. Defining the error function as $e(x)=u(x)-u_{h}(x)$ it is a simple algebrism to prove that $e\left(x_{i}\right)=0$, hence, one follows from the Mean Value Theorem that there is at least one point $\bar{\xi}$ inside $\widehat{I}$ so that $e^{\prime}(\bar{\xi})=0$. Starting from this fact, one can 
interpret the existence of points where the convergence order for derivatives is higher than the usual one. This observation was done by Strang and Fix [12] in the sense of a purely mathematical interpolation problem. What exactly means that the true field $u$ and the interpolated field $u_{h}$ are such that in certain notable points $\bar{\xi}$

$$
u^{\prime}(\bar{\xi})=u_{h}^{\prime}(\bar{\xi}) .
$$

Naturally, such kind of coincidence depends on the functional space to which the interpolant and exact field belong to. Several arguments and paradigms have been applied to overcome the fundamental problem in the determination of the superconvergent points for derivatives. The central point in this topic is summarized by the fact that the exact solution is unknown or equivalently the functional space to which $u$ belongs to is not known as well. To overcome this, Prathap's paradigm assumes explicitly that the true field $u \in P_{k+1}(\widehat{I})$, therefore, $u$ is a one polynomial order higher than the lagrangian finite element interpolant $u_{h}$. This hypothesis was also assumed by MacNeal to determine the Barlow Points and to demonstrate that in general Gaussian quadrature rule points do not coincide with the former (see also recent paper by Zhang [16]). The Gaussian quadrature points are defined as the zeros of the Legendre's Polinomials on $[-1,+1]$ (see Table 1$)$.

\begin{tabular}{|c|l|}
\hline order $n$ & $P_{n}(\xi)$ \\
\hline 1 & $2 \xi$ \\
\hline 2 & $4\left(3 \xi^{2}-1\right)$ \\
\hline 3 & $24\left(5 \xi^{3}-3 \xi\right)$ \\
\hline 4 & $48\left(35 \xi^{4}-30 \xi^{2}+3\right)$ \\
\hline 5 & $480\left(63 \xi^{5}-70 \xi^{3}+15 \xi\right)$ \\
\hline
\end{tabular}

Table 1 - Gauss-Legendre's Polynomial.

\section{Definition of superconvergence}

Typically, if the error in the finite element field converges as $h^{k+1}$, then, the error in the finite element gradient field converges as $h^{k}$ where $h$ is the maximum diameter 
of the finite element discretization and $k$ is the spectral order of the interpolation. These facts are well known in the classical finite element interpolation theory and given by the classical estimates in $L^{2}$-norm

$$
\begin{aligned}
& \left\|u-u_{h}\right\|_{L^{2}} \leq C h^{k+1} \\
& \left\|u^{\prime}-u_{h}^{\prime}\right\|_{L^{2}} \leq C h^{k}
\end{aligned}
$$

Generally speaking, the superconvergence is defined as a higher order of convergence which is exhibited by interior points of the finite element solution where the derivative assumes an $O\left(h^{k+1}\right)$ convergence, thus, one order higher when compared with an $O\left(h^{k}\right)$ of the finite element derivative field. Historically, the higher order accuracy property was first noticed by Barlow [1] and its existence was firstly formulated by Strand and Fix [12]. This concept can be extended to similar properties but referring to $O\left(h^{k+2}\right)$ convergence and $O\left(h^{k+3}\right)$ convergence called simply ultraconvergence and hyperconvergence by using systematically the Taylor's Series Theorem $[5,6]$. The superconvergence is also linked to the concept of reduced integration and zero energy modes so important in the Finite Element Theory [15]. Even more important, the superconvergence points are of utility as sampling points for the finite element designers in formulations like gradient smoothening or gradient recovery in the sense of The Superconvergent Patch Recovery as sketched by Zienkiewcz [13]. However, the major significance of these points that explains all kind of numerical and analytical studies in this topic refers to its application in adaptive finite element analysis, especially for construction of error indicators based on the recovered gradient or hessian.

\section{The Taylor's series expansion theorem}

Limiting the discussion to a one dimensional problem for a discretization with equally spaced nodes and using a lagrangian basis, the finite element interpolant for a given exact function $u$ is written simply as

$$
u_{h}(x)=\sum_{i=0}^{k} u_{i} L_{i}(x),
$$


where $u_{i}=u\left(x_{i}\right)$ is the degree of freedom calculated in the nodal point $x_{i}$. The functional degree of freedom $u_{i}$ is, then, expanded around a point $\bar{x}$ inside the element by applying the Taylor's series expansion which can be expressed as

$$
u\left(x_{i}\right)=\sum_{i=0}^{\infty} \frac{1}{i !} D_{x}^{i} u(\bar{x}) h_{x}^{i}
$$

where $h_{x}^{i}=x_{i}-\bar{x}$ and $D_{x}^{i}=\frac{d^{i}}{d x^{i}}$.

Under these conditions the interpolant $u_{h}$ can be formally differentiated to form

$$
u_{h}^{\prime}(x)=\sum_{i=0}^{k} u_{i} L_{i}^{\prime}(x),
$$

The functional degree of freedom is, then, expanded around an arbitrary point $\bar{x}$ inside the element by applying the Taylor's Series Expansion and, hence, the derivative field $u_{h}$ is written in the form

$$
u_{h}^{\prime}(x)=\sum_{i=0}^{k} L_{i}^{\prime}(x)\left(\sum_{j=0}^{\infty} \frac{1}{j !} D_{x}^{j} u(\bar{x}) h_{x_{i}}^{j},\right),
$$

where $h_{x_{i}}=x_{i}-\bar{x}$.

Rearranging the terms, $u_{h}^{\prime}(x)=\sum_{i=0}^{k} u_{i} L_{i}^{\prime}(x)$ can be expressed as

$$
\begin{aligned}
u_{h}^{\prime}(x)= & \left(\sum_{i=1}^{N} L_{i}^{\prime}(x)\right) u(x)+\left(\sum_{i=1}^{N} L_{i}^{\prime}\left(x_{i}-\bar{x}\right)\right) u^{\prime}(x) \\
& +\left(\sum_{i=1}^{N} \frac{L_{i}^{\prime}(\bar{x})\left(x_{i}-\bar{x}\right)^{2}}{2 !}\right) u^{\prime \prime}(\bar{x})+\ldots
\end{aligned}
$$

After deriving the partition unity identity for Lagrange's Shape Function Basis one obtains

$$
u^{\prime}(\bar{x})-\sum_{i=1}^{N} u_{i} L_{i}^{\prime}(\bar{x})=-\sum_{j=2}^{\infty}\left(\sum_{i=1}^{N} \frac{L_{i}^{\prime}(\bar{x})\left(x_{i}-\bar{x}\right)^{j}}{j !}\right) u^{(j)}(\bar{x})
$$

where

$$
\sum_{i=1}^{N} L_{i}^{\prime}(x)=0
$$


Defining the exact error in the derivative field as $e^{\prime}(\bar{x})=\left|u^{\prime}(\bar{x})-u_{h}^{\prime}(\bar{x})\right|$ and noting that all terms vanish until the order $k$ by applying the completeness properties of Lagrange's Basis then [3]

$$
e^{\prime}(\bar{x})=\left|-\sum_{j=k+1}^{\infty}\left(\sum_{i=1}^{N} \frac{L_{i}^{\prime}(\bar{x})\left(x_{i}-\bar{x}\right)^{j}}{j !} u^{(j)}(\bar{x})\right)\right| .
$$

By using the generalized triangular inequality, the error expression $e^{\prime}(\bar{x})$ allows us to define a consistent superconvergence concept by using the equivalence binary relation

$$
\alpha_{-} O\left(h^{p}\right) \leq f(\bar{x}) \leq \alpha_{+} O\left(h^{p}\right), \alpha_{-}, \alpha_{+} \in R^{+} \Leftrightarrow f=O\left(h^{p}\right),
$$

$p \in N$ is the spectral order of the lagrangian element. Hence, the existence of superconvergent points for the first order derivative consist of the points $\bar{x}$ inside the element where the exact error expression satisfies the superconvergence condition given by $\left|u^{\prime}(\bar{x})-u_{h}^{\prime}(\bar{x})\right|=O\left(h^{k+1}\right)$.

\subsection{Definition of superconvergent points}

By a superconvergent point $\bar{x}$ for $u_{h}^{\prime}$, one understands a point such as

$$
s_{k}(x)=\sum_{i=0}^{k} \frac{L_{i}^{\prime}(x)\left(x_{i}-x\right)^{k+1}}{(k+1) !}=0 \Leftrightarrow e^{\prime}(\bar{x})=O\left(h^{k+1}\right) .
$$

The function $s_{k}(x)$ will be hereinafter referred to as superconvergence function or zero-error mode for a derivative field $u^{\prime}(x) h$. Similarly, one can define the ultraconvergent points and hyperconvergent points for the derivative field $u_{h}^{\prime}$.

\subsection{Definition of ultraconvergent points}

By a ultraconvergent point $\bar{x}$ for $u_{h}^{\prime}$, one understands a point such as

$$
\left\{\begin{array}{l}
s_{k}(x)=\sum_{i=0}^{k} \frac{L_{i}^{\prime}(x)\left(x_{i}-x\right)^{k+1}}{(k+1) !}=0, \\
u_{k}(x)=\sum_{i=0}^{k} \frac{L_{i}^{\prime}(x)\left(x_{i}-x\right)^{k+2}}{(k+2) !}=0
\end{array} \Leftrightarrow e^{\prime}(\bar{x})=O\left(h^{k+2}\right) .\right.
$$




\subsection{Definition of hyperconvergent points}

By a ultraconvergent point $\bar{x}$ for $u_{h}^{\prime}$, one understands a point such as

$$
\left\{\begin{array}{l}
s_{k}(x)=\sum_{i=0}^{k} \frac{L_{i}^{\prime}(x)\left(x_{i}-x\right)^{k+1}}{(k+1) !}=0, \\
u_{k}(x)=\sum_{i=0}^{k} \frac{L_{i}^{\prime}(x)\left(x_{i}-x\right)^{k+2}}{(k+2) !}=0, \quad \Leftrightarrow e^{\prime}(\bar{x})=O\left(h^{k+3}\right) . \\
h_{k}(x)=\sum_{i=0}^{k} \frac{L_{i}^{\prime}(x)\left(x_{i}-x\right)^{k+3}}{(k+3) !}=0
\end{array}\right.
$$

Hence, starting from a Lagrange's Family element interpolant which derivative is calculated by differentiating the interpolant, a set of superconvergent points for the first order derivative is, then, obtained by calculating the zeros of the superconvergence function $s_{k}(x)$. Interestingly, the superconvergence function $s_{k}(x)$ can be expressed simply as the following polynomial

$$
s_{k}(x)=\sum_{i=0}^{k} L_{i}^{\prime}(x) x_{i}^{k+1}-(k+1) x^{k}=0
$$

after using all completeness properties of Lagrange's basis, where $\left\{L_{i}\right\}_{i=0}^{k}$ is the Lagrange's Basis, $k$ is the spectral order of the interpolation and $x$ is the coordinate of an arbitrary point inside the reference element. If the symbolic software, Mathematica, is used a remarkable set of points can be calculated analytically which are the zeros of Legendre polynomials and, hence, the Gaussian quadrature points but not for higher spectral order as inferred from Table 1. It's important to emphasize that the completeness property of the Lagrange's basis is fundamental in order to simplify the deduction by applying the ideas firstly discussed by Carey [3]. Basically, the completeness consists of several identities that depend on the degree of lagrangian basis function. The fundamental expression in terms of completeness property represents the partition of unity and is written as

$$
\sum_{i=0}^{k} L_{i}(x)=1 .
$$

Similarly, expressions like the previous one can be proved but their number restricts their citation (see reference [3] and references therein). 
The superconvergence curve $s_{k}(x)$ is plotted against the x-coordinate in Figure 1 for $k=1,2,3,4,5$ over the interval $[-1,+1]$. In each case, the curve $s_{k}$ intersects the $x$-axes $k$ times at the superconvergent points where the $e^{\prime}(\bar{x})=$ $O\left(h^{k+1}\right)$. The zero-error points for the derivative field are summarized in Table 1. Similarly, the Gauss-Legendre polynomials $P_{n}(x)$ are plotted against the $x$-coordinate in Figure 2 for $n=1,2,3,4,5$. It is then obvious that, by comparing Figure 1 and Figure 2, the fact notified by Prathap that the Gauss points may or may not coincide with the Barlow points especially for higher order elements. Comparison of Figure 1 and Figure 2 shows that the curves $s_{k}(x)$ and $P_{n}(x)$ are similar qualitatively both in shape and number of zeros whenever $k=n$ although significantly different in terms of quantification as observed from the algebraic expressions for $s_{k}(x)$ and $P_{n}(x)$, summarized in Table 1 and Table 2. The ultraconvergence functions $u_{k}(x)$ for the derivative field are listed in Table 3. These functions do not coincide with the superconvergence functions for the case of $k=1,2,3,4,5$ as summarized in Table 2. By comparing the zeros of $u_{k}$ and $s_{k}$ (see Table 4 and Table 5) one can conclude that an $O\left(h^{k+2}\right)$ convergence is impossible to happen mathematically with a straightforward application of the Taylor's Series paradigm. Therefore, the ultraconvergence phenomenon as notified by Zienkiewicz in his numerical experiments are believed to be a numerical coincidence (see reference [13]). It is important to comment the existence of purely imaginary ultraconvergent zeros as one can infer from Table 4. It is not a simple matter of interpreting the significance of such kind of points in terms of numerical convergence. Note also that the centroid is a ultraconvergent point for quadratic, cubic and quartic lagrangian elements, unlike in the case of $k=1$ and $k=5$. This can explain consistently the usual choice of the geometric center of the element as the natural sampling point for the calculation of the gradient finite element designers.

\section{Superconvergence invariance}

Let $x=G(\xi)$ be a geometric transformation from the reference element onto a lagrangian finite element with nodes $x_{0}, x_{1}, \cdots, x_{k}$. Assuming that the set of nodes is equally spaced the isoparametric mapping $x=G(\xi)$ is defined 
a)

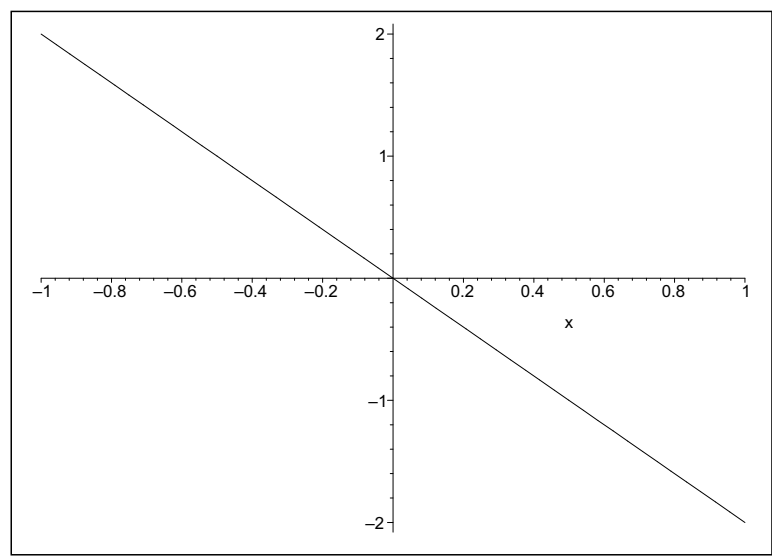

b)

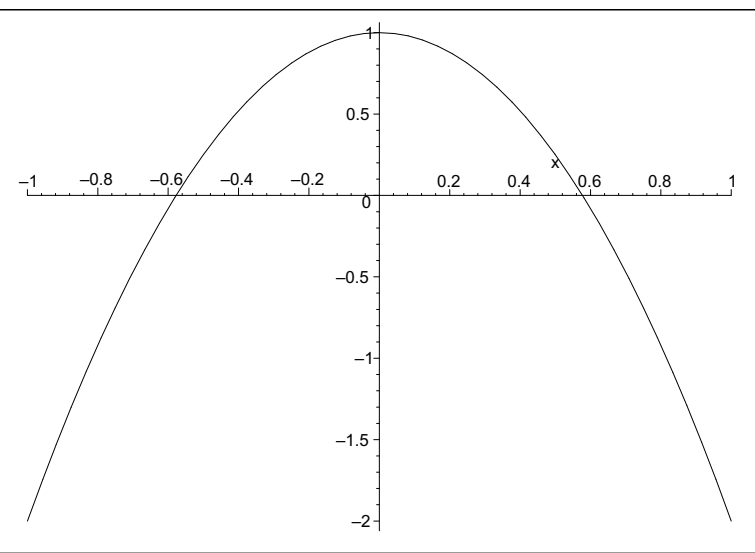

c)

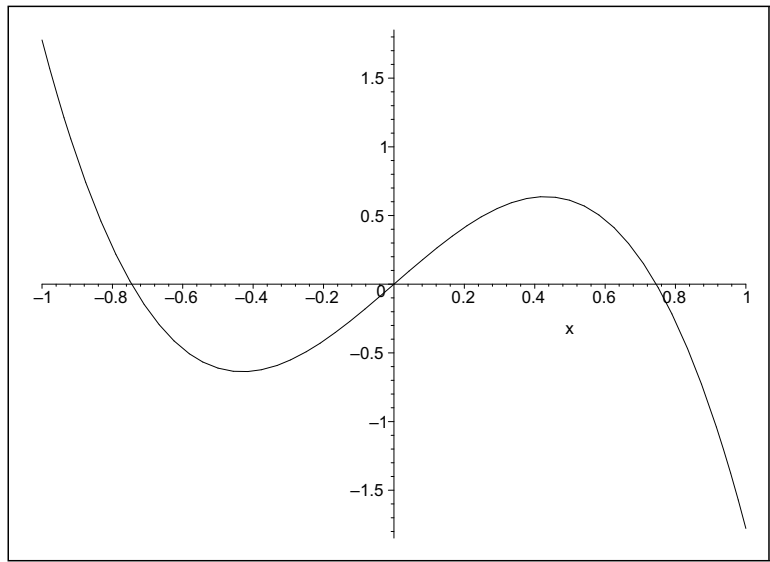

Figure $1(\mathrm{a}, \mathrm{b}, \mathrm{c})$ - Zero-error modes $s_{k}(x)$ for $u_{h}^{\prime}(x), k=1,2,3,4,5$. 
d)

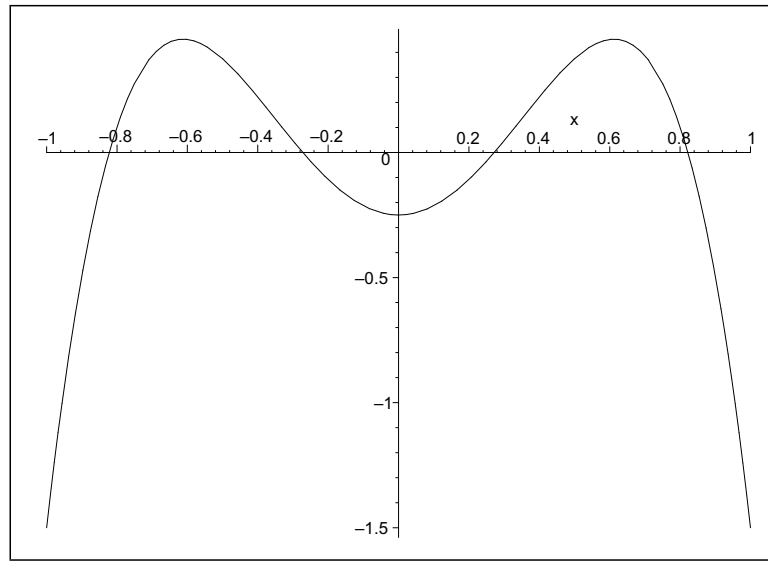

e)

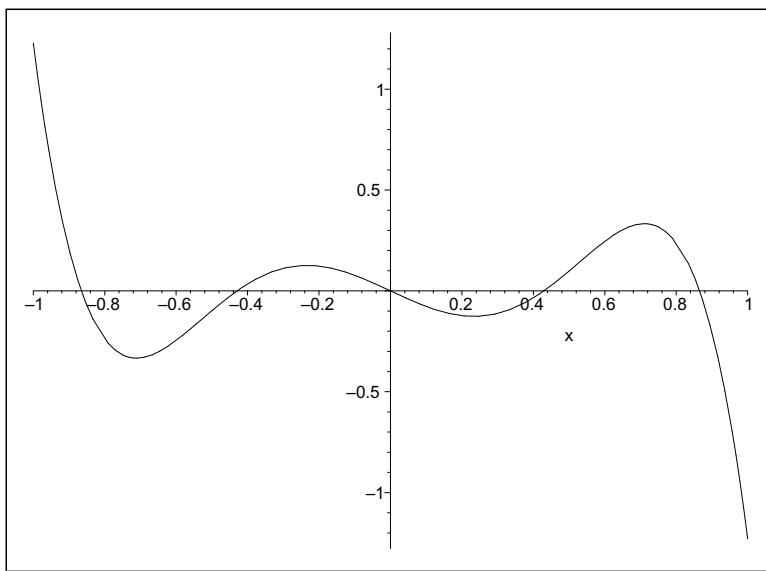

Figure $1(\mathrm{~d}, \mathrm{e})$ - Zero-error modes $s_{k}(x)$ for $u_{h}^{\prime}(x), k=1,2,3,4,5$.

\begin{tabular}{|c|l|}
\hline order $k$ & $s_{k}(x)$ \\
\hline 1 & $-2 x$ \\
\hline 2 & $-3 x^{2}+1$ \\
\hline 3 & $-4 x^{3}+\frac{20}{9} x$ \\
\hline 4 & $-5 x^{4}+\frac{15}{4} x^{2}-\frac{1}{4}$ \\
\hline 5 & $-6 x^{5}+\frac{28}{5} x^{3}-\frac{518}{625} x$ \\
\hline
\end{tabular}

Table 2 - Zero-error modes for $u_{h}^{\prime}$. 
a)

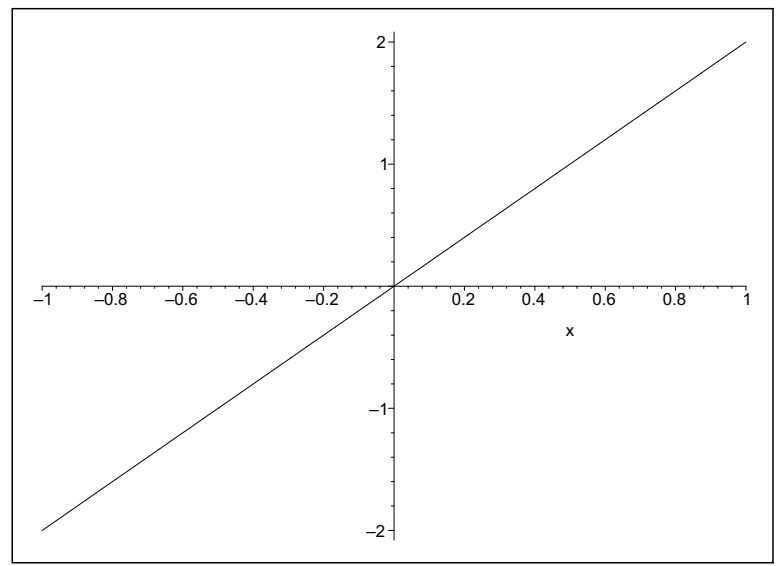

b)

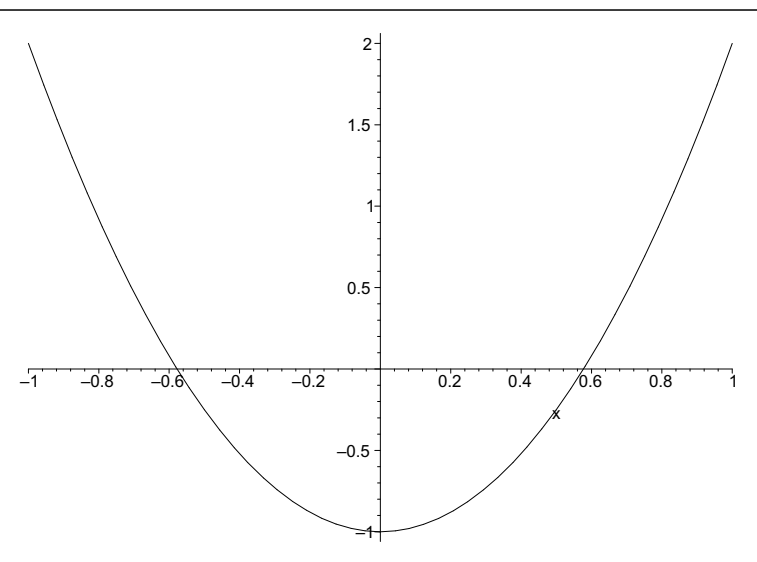

c)

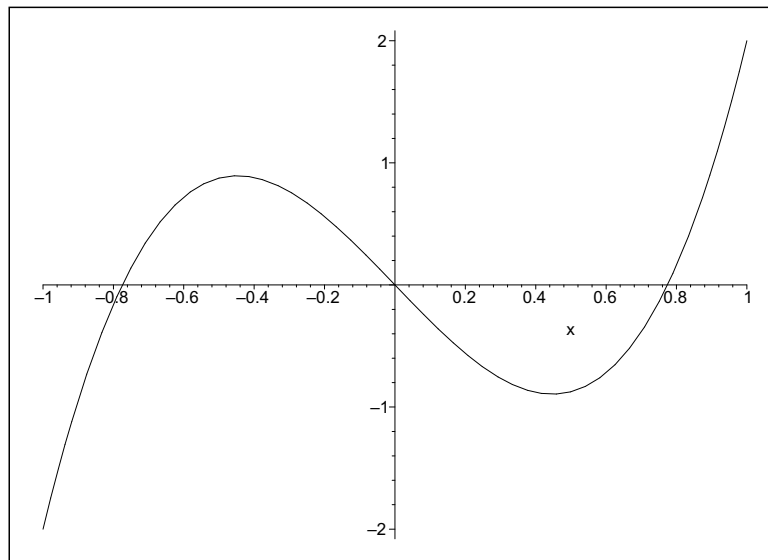

Figure 2 (a, b, c) - Gauss-Legendre's Polynomials $P_{n}(x)$ for $n=1,2,3,4,5$. 
d)

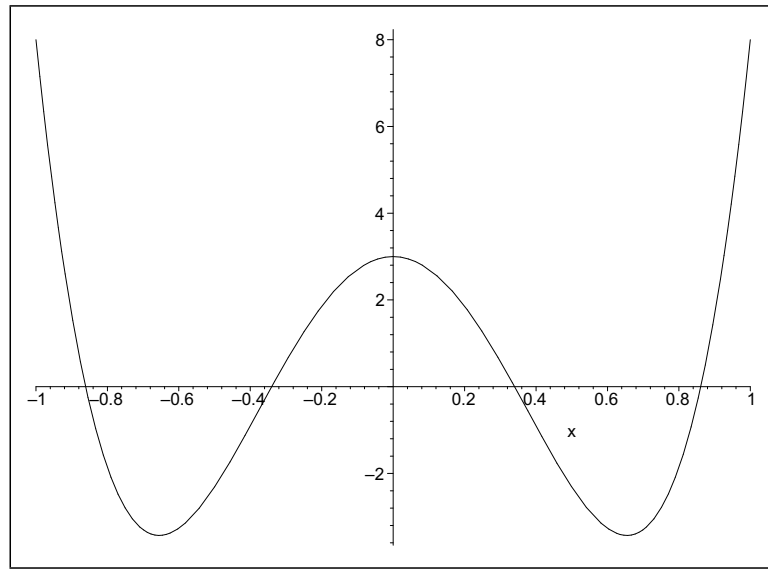

e)

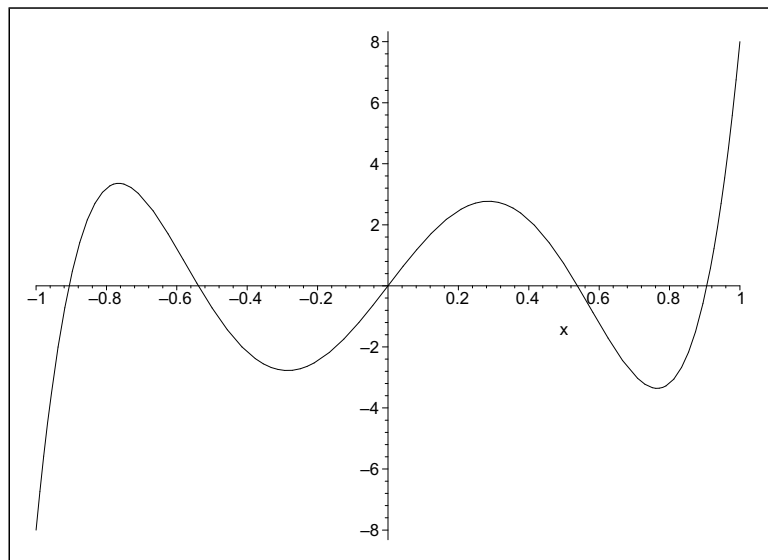

Figure $2(\mathrm{~d}, \mathrm{e})$ - Gauss-Legendre's Polynomials $P_{n}(x)$ for $n=1,2,3,4,5$.

\begin{tabular}{|c|l|}
\hline order $k$ & $u_{k}(x)$ \\
\hline 1 & $1+3 x^{2}$ \\
\hline 2 & $-2 x+8 x^{3}$ \\
\hline 3 & $-\frac{1}{9}-\frac{70}{9} x^{2}+15 x^{4}$ \\
\hline 4 & $x-\frac{35}{2} x^{3}+24 x^{5}$ \\
\hline 5 & $\frac{9+2849 x^{2}-20125 x^{4}+21875 x^{6}}{625}$ \\
\hline
\end{tabular}

Table 3 - Ultraconvergence function for $u_{h}^{\prime}$. 


\begin{tabular}{|l|l|}
\hline $\mathrm{k}$ & Ultraconvergent points \\
\hline 1 & $\pm \frac{\sqrt{3}}{3} i$ \\
\hline 2 & $0, \pm \frac{1}{2}$ \\
\hline 3 & $0, \pm \frac{\sqrt{7+4 \sqrt{\frac{17}{5}}}}{3 \sqrt{3}}= \pm 0.72967, \pm \frac{\sqrt{7-4 \sqrt{\frac{17}{5}}}}{3 \sqrt{3}}= \pm 0.11795 i$ \\
\hline 4 & $0, \pm \frac{1}{4}, \pm \sqrt{\frac{2}{3}}$ \\
\hline 5 & $\pm 0.86270 \pm 3.10^{-18} i, \pm 0.42287 \pm 5.10^{-17} i, \pm 4.10^{-16} \pm 0.05559 i$ \\
\hline
\end{tabular}

Table $4-$ Zeros of the ultraconvergence function for $u_{h}^{\prime}$.

\begin{tabular}{|l|l|l|}
\hline $\mathrm{k}$ & Superconvergent points & Gauss points \\
\hline 1 & 0 & 0 \\
\hline 2 & $\pm \sqrt{3} / 3= \pm 0.57735$ & $\pm \sqrt{3} / 3= \pm 0.57735$ \\
\hline 3 & $0, \pm \sqrt{5} / 3= \pm 0.74535$ & $0, \pm \sqrt{3 / 5}= \pm 0.77459$ \\
\hline 4 & $\pm \frac{\sqrt{3 \pm \sqrt{29 / 5}}}{2 \sqrt{2}}= \pm 0.27195, \pm 0.82221$ & $\pm \sqrt{\frac{3}{7} \pm \frac{2 \sqrt{6}}{7 \sqrt{5}}}= \pm 0.33998, \pm 0.861136$ \\
\hline 5 & $0, \pm \frac{\sqrt{35 \pm 8 \sqrt{7}}}{5 \sqrt{3}}= \pm 0.42948, \pm 0.86537$ & $0, \pm \frac{\sqrt{5 \pm 2 \sqrt{10 / 7}}}{3}= \pm 0.53846, \pm 0.90617$ \\
\hline
\end{tabular}

Table 5 - Numerical values of superconvergent points and Gauss points.

simply as

$$
x=G(\xi)=\sum_{i=1}^{k} L_{i}(\xi) x_{i}
$$

By a simple algebrism one can express the transformation above for a linear lagrangian element as $x=\frac{x_{0}+x_{1}}{2}+\frac{x_{1}-x_{0}}{2} \xi$. Similarly, for a quadratic lagrangian element the mapping becomes simply $x=x_{1}+\frac{x_{2}-x_{0}}{2} \xi+\frac{x_{0}-2 x_{1}+x_{2}}{2} \xi^{2}$. Noting that by hypothesis the nodes are equally spaced then the quadratic term vanishes and thus, for $k=2, x=x_{1}+\frac{x_{2}-x_{0}}{2} \xi$. Analogously, for $k=3$ the isoparametric transformation is expressed as

$$
\begin{aligned}
x= & \frac{-x_{0}+9 x_{1}+92-x_{3}}{16}+\frac{x_{0}-27 x_{1}+27 x_{2}-x_{3}}{16} \xi \\
& +\frac{9\left(x_{0}-x_{1}-x_{2}+x_{3}\right)}{16} \xi^{2}+\frac{-9 x_{0}+27 x_{1}-27 x_{2}+9 x_{3}}{16} \xi^{3} .
\end{aligned}
$$


Hence, after applying the assumption of equally spaced nodes within a cubic element, one obtains

$$
x=\frac{x_{0}+x_{3}}{2}+\frac{x_{3}-x_{0}}{2} \xi .
$$

For a quartic lagrangian element the isoparametric mapping is of the form

$$
\begin{aligned}
x= & x_{2}+\frac{x_{0}-8 x_{1}+8 x_{3}-x_{4}}{6} \xi+\frac{-x_{0}+16 x_{1}-30 x_{2}+16 x_{3}-x_{4}}{6} \xi^{2} \\
& +\frac{-4 x_{0}+8 x_{1}-8 x_{3}+4 x_{3}}{6} \xi^{3}+\frac{4 x_{0}-16 x_{1}+24 x_{2}-16 x_{3}+4 x_{4}}{6} \xi^{4} .
\end{aligned}
$$

Again, with a uniform distribution of nodes within the element, the isoparametric transformation can be expressed as

$$
x=\frac{x_{0}+x_{4}}{2}+\frac{x_{4}-x_{0}}{2} \xi .
$$

The explicit analytical expression for the isoparametric mapping corresponding to a quintic lagrangian element can also be deduced which is represented by the polynomial $x=a_{0}+a_{1} \xi+a_{2} \xi^{2}+a_{3} \xi^{3}+a_{4} \xi^{4}+a_{5} \xi^{5}$ where the coefficients are

$$
\left\{\begin{array}{l}
a_{0}=\frac{9 x_{0}-75 x_{1}+450 x_{2}+450 x_{3}-75 x_{4}+9 x_{5}}{768}, \\
a_{1}=\frac{-9 x_{0}+125 x_{1}-2250 x_{2}+2250 x_{3}-125 x_{4}+9 x_{5}}{768}, \\
a_{2}=\frac{-250 x_{0}+1950 x_{1}-1700 x_{2}-1700 x_{3}+1950 x_{4}-250 x_{5}}{768}=0, \\
a_{3}=\frac{250 x_{0}-3250 x 1+8500 x_{2}-8500 x_{3}+3250 x_{4}-250 x_{5}}{768}=0 \\
a_{4}=\frac{625 x_{0}-1875 x_{1}+1250 x_{2}+1250 x_{3}-1875 x_{4}+625 x_{5}}{768}=0 \\
a_{5}=\frac{-625 x_{0}+3125 x_{1}-6250 x_{2}+6250 x_{3}-3125 x_{4}+625 x_{5}}{768}=0 .
\end{array}\right.
$$

It's simple to verify that the coefficients of higher degree are identically null under the nodal uniformity condition, thus, the mapping above has its linear part reduced to the affine map

$$
x=\frac{x_{0}+x_{5}}{2}+\frac{x_{5}-x_{0}}{2} \xi .
$$

Starting from a uniformity condition of nodal distribution inside the one dimensional lagrangian element, having nodal points $x_{i}=x_{0}+i h, i=1, \ldots, k$, 
where $h=x_{i}-x_{i-1}$ and after expanding

$$
x=G(\xi)=\sum_{i=1}^{k} L_{i}(\xi) x_{i}
$$

one can prove that all higher degree, nonlinear terms vanish. Generalizing by induction the isoparametric mapping is simply the affine mapping

$$
x=\bar{x}+J_{x} \xi
$$

where $\bar{x}$ is the geometric centroid of the element, $J_{x}=h_{x} / 2$ is the jacobian of the transformation and $h_{x}$ is the length of the element.

Therefore, if equally spaced nodes are used the isoparametric mapping can be expressed as an affine transformation which is obviously bijective and thus invertible. In this particular case, the set of superconvergent points $S_{k, \hat{I}}=$ $\left\{\xi_{i} \in \hat{I} \mid s_{k}\left(\xi_{i}\right)=0,1 \leq i \leq k\right\}$ is such that card $S_{k, \hat{I}}=k=\operatorname{card}\left(G\left(S_{k, \hat{I}}\right)\right.$. In conclusion, whenever the nodes are uniformly distributed the isoparametric mapping is an affine transformation and under this condition the number of zeros for $s_{k}(x)$ is equal to those for $\left(s_{k} \circ G^{-1}\right)(x)$. It means that the superconvergent points are preserved on a general uniform element.

In this section, we analyse the question of the invariance of the superconvergence properties if the nodal points are not equally spaced inside the element. In order to study the variation of the superconvergent coordinates inside the reference element a single node can move freely while all remainders are kept on fixed.

The consistence of formulas can also be applied to explain the coincidence between the Barlow points and Gaussian quadrature points for linear elements in $1 \mathrm{D}$ with nodes $x_{0}$ and $x_{1}$. In this particular case, the superconvergent points are simply the zero of the superconvergence function

$$
s_{1}(x)=-2 x+x_{0}+x_{1}=0 .
$$

Obviously, the zero is the centroid of the element which coincides exactly with the Gauss quadrature point obtained by calculating the zero of the Legendre's polynomial over the reference element $[-1,+1]$. One can reproduce exactly 
the superconvergence function for a two node element by comparing the former with the Legendre's polynomial written in the form

$$
P_{n}(x)=D_{x}^{n}\left[\left(x^{2}-1\right)^{n}\right], D_{x}^{n}=\frac{d^{n}}{d x^{n}},
$$

for $n=0$ and determining the zeros of $P_{n+1}(x)=P_{0+1}(x)=D_{x}^{1}\left[\left(x^{2}-1\right)^{1}\right]$.

Let us consider a three node one dimensional element. Assuming that the midpoint $x_{1}$ is free the superconvergence function $s_{2}(x)$ is written as

$$
s_{2}(x)=a_{2}\left(x_{1}\right) x^{2}+a_{1}\left(x_{1}\right) x+a_{0}\left(x_{1}\right)=0 .
$$

If the nodal points are equally spaced inside the element this polynomial coincides with the Legendre polynomial where $a_{2}\left(x_{1}\right)=3, a_{1}\left(x_{1}\right)=0, a_{0}\left(x_{1}\right)=$ -1 , which zeros are the Gaussian quadrature points. However, if the midpoint is randomly positioned inside the element, then, the superconvergence function is

$$
s_{2}(x)=3 x^{2}+2 x_{1} x-1=0 .
$$

Therefore, the polynomial coefficients are not invariant under a coordinate changing of the nodal points. Such counter-example shows clearly that the superconvergent points position may vary inside the element for an arbitrary element with randomly distributed nodes.

Similar calculations can be performed for a four nodes one dimensional element by assuming, without limitation of generality, that $x_{1}$ and $x_{2}$ are varying freely under the symmetric configuration of nodes expressed as $x_{1}=-x_{2}$ while the end nodes are fixed and set to be $x_{0}=-1$ and $x_{3}=+1$. In this case, the superconvergence function is written in the form

$$
s_{3}(x)=2 x^{3}-\left(1+x_{1}^{2}\right) x=0 .
$$

By comparing the superconvergence function $s_{3}(x)$ for a cubic element with a Legendre's polynomial of third degree which zeros are calculated from $5 x^{3}-$ $3 x=0$, one can infer that the zeros of $s_{3}(x)$ reproduce exactly the Barlow points, setting $x_{1}=-x_{2}=-1 / 3$ as illustrated in Table 1 .

Let us now assume that the nodal configuration inside a five nodes one dimensional element permits $x_{1}$ and $x_{3}$ vary freely while $x_{0}=-1, x_{2}=0$ and 
$x_{4}=+1$. To simplify the algebrism, it is assumed that $x_{1}$ and $x_{3}$ are symmetrically placed, thus, $x_{1}=-x_{3}$. After substituting all completeness properties for a quartic element and the imposed symmetry condition, the superconvergence function up to a signal is written as

$$
s_{4}(x)=5 x^{4}+\left[-3\left(1+x_{1}^{2}\right)\right] x^{2}+x_{1}^{2}=0 .
$$

For the purpose of expressing the superconvergence function $s_{5}(x)$ in terms of a single free node let us enforce the symmetry condition $x_{2}=-x_{3}$ for a six nodes lagrangian finite element. Using this condition, the $s_{5}(x)$ becomes

$$
s_{5}(x)=-6 x^{5}+\left(\frac{136}{25}+4 x_{2}^{2}\right) x^{3}-\frac{\left(18+68 x_{2}\right)}{25} x=0 .
$$

Firstly, this confirms one more time that the coefficients depend on, in general, the nodal coordinates. In conclusion, the superconvergence function is not invariant for a configuration of randomly distributed nodes inside the element.

In fact, MacNeal [4] found out that Gaussian quadrature points and Barlow points are coincident for lower order elements whereas it is not true for higher order elements as illustrated in Table 1. Similarly, Prathap [10] showed how to determine the Barlow points from a variational basis and from the Taylor's Series approach it is also possible to work out that for not equally spaced nodes inside the element, in general, the superconvergence point can change its coordinate.

\section{The Hermitian Basis}

For the purpose of calculating optimal points $\bar{x}$ in case of a Hermitian Basis $H_{i}(x), i=0,1,2,3$, the hermitian interpolant is assumed to be the linear combination

$$
u_{h}(x)=u_{0} H_{0}(x)+u_{1} H_{1}(x)+u_{0}^{\prime} H_{2}(x)+u_{1}^{\prime} H_{3}(x) .
$$

By deriving the equation (29), one follows that

$$
u_{h}^{\prime}(\bar{x})=u\left(x_{0}\right) H_{0}^{\prime}(\bar{x})+u\left(x_{1}\right) H_{1}^{\prime}(\bar{x})+u^{\prime}\left(x_{0}\right) H_{2}^{\prime}(\bar{x})+u^{\prime}\left(x_{1}\right) H_{3}^{\prime}(\bar{x})
$$

Expanding the degree of freedom $u_{0}, u_{1}, u_{0}^{\prime}, u_{1}^{\prime}$ with $h_{x_{0}}=x_{0}-\bar{x} \mathrm{e}$ $h_{x_{1}}=x_{1}-\bar{x}$ around the $\bar{x}$ one obtains

$$
u_{0}=u\left(x_{0}\right)=u(\bar{x})+u^{\prime}(\bar{x}) h_{x_{0}}+u^{\prime \prime}(\bar{x}) \frac{h_{x_{0}}^{2}}{2 !}+u^{(3)} \frac{h_{x_{0}}^{3}}{3 !}+u^{(4)}(\bar{x}) \frac{h_{x_{0}}^{4}}{4 !}+\cdots
$$




$$
\begin{aligned}
& u_{1}=u\left(x_{1}\right)=u(\bar{x})+u^{\prime}(\bar{x}) h_{x_{1}}+u^{\prime \prime}(\bar{x}) \frac{h_{x_{1}}^{2}}{2 !}+u^{(3)}(\bar{x}) \frac{h_{x_{1}}^{3}}{3 !}+u^{(4)}(\bar{x}) \frac{h_{x_{1}}^{4}}{4 !}+\cdots \\
& u_{0}^{\prime}=u^{\prime}\left(x_{0}\right)=u^{\prime}(\bar{x})+u^{\prime \prime}(\bar{x}) h_{x_{0}}+u^{(3)}(\bar{x}) \frac{h_{x_{0}}^{2}}{2 !}+u^{(4)}(\bar{x}) \frac{h_{x_{0}}^{3}}{3 !}+\cdots \\
& u_{1}^{\prime}=u^{\prime}\left(x_{1}\right)=u^{\prime}(\bar{x})+u^{\prime \prime}(\bar{x}) h_{x_{1}}+u^{(3)}(\bar{x}) \frac{h_{x_{1}}^{2}}{2 !}+u^{(4)}(\bar{x}) \frac{h_{x_{1}}^{3}}{3 !}+\cdots
\end{aligned}
$$

After substituting equations (31), (32), (33), (34) in equation (29), $u_{h}^{\prime}(x)$ takes the form

$$
\begin{aligned}
u_{h}^{\prime}(\bar{x})= & {\left[u(\bar{x})+u^{\prime}(\bar{x}) h_{x_{0}}+u^{\prime \prime}(\bar{x}) \frac{h_{x_{0}}^{2}}{2 !}+u^{(3)}(\bar{x}) \frac{h_{x_{0}}^{3}}{3 !}+u^{(4)}(\bar{x}) \frac{h_{x_{0}}^{4}}{4 !}+\cdots\right] H_{0}^{\prime}(\bar{x}) } \\
& +\left[u(\bar{x})+u^{\prime}(\bar{x}) h_{x_{1}}+u^{\prime \prime}(\bar{x}) \frac{h_{x_{1}}^{2}}{2 !}+u^{(3)}(\bar{x}) \frac{h_{x_{1}}^{3}}{3 !}+u^{(4)}(\bar{x}) \frac{h_{x_{1}}^{4}}{4 !}+\cdots\right] H_{1}^{\prime}(\bar{x}) \\
& +\left[u^{\prime}(\bar{x})+u^{\prime \prime}(\bar{x}) h_{x_{0}}+u^{(3)}(\bar{x}) \frac{h_{x_{0}}^{2}}{2 !}+u^{(4)}(\bar{x}) \frac{h_{x_{0}}^{3}}{3 !}+\cdots\right] H_{2}^{\prime}(\bar{x}) \\
& +\left[u^{\prime}(\bar{x})+u^{\prime \prime}(\bar{x}) h_{x_{1}}+u^{(3)}(\bar{x}) \frac{h_{x_{1}}^{2}}{2 !}+u^{(4)}(\bar{x}) \frac{h_{x_{1}}^{3}}{3 !}+\cdots\right] H_{3}^{\prime}(\bar{x})
\end{aligned}
$$

Rearranging the terms in the equation (35), the resulting equation is then

$$
\begin{aligned}
u_{h}^{\prime}(\bar{x})= & \underbrace{\left[H_{0}^{\prime}(\bar{x})+H_{1}^{\prime}(\bar{x})\right]}_{I_{1}} u(\bar{x}) \\
& +\underbrace{\left[H_{0}^{\prime}(\bar{x}) h_{x_{0}}+H_{1}^{\prime}(\bar{x}) h_{x_{1}}+H_{2}^{\prime}(\bar{x})+H_{3}^{\prime}(\bar{x})\right]}_{I_{2}} u^{\prime}(\bar{x}) \\
& +\underbrace{\left[H_{0}^{\prime}(\bar{x}) \frac{h_{x_{0}}^{2}}{2 !}+H_{1}^{\prime}(\bar{x}) \frac{h_{x_{1}}^{2}}{2 !}+H_{2}^{\prime}(\bar{x}) h_{x_{0}}+H_{3}^{\prime}(\bar{x}) h_{x_{1}}\right]}_{I_{3}} u^{\prime \prime}(\bar{x}) \\
& +\underbrace{\left[H_{0}^{\prime}(\bar{x}) \frac{h_{x_{0}}^{3}}{3 !}+H_{1}^{\prime}(\bar{x}) \frac{h_{x_{1}}^{3}}{3 !}+H_{2}^{\prime}(\bar{x}) \frac{h_{x_{0}}^{2}}{2 !}+H_{3}^{\prime}(\bar{x}) \frac{h_{x_{1}}^{2}}{2 !}\right]}_{I_{5}} u^{(3)}(\bar{x}) \\
& +\underbrace{\left[H_{0}^{\prime}(\bar{x}) \frac{h_{x_{0}}^{4}}{4 !}+H_{1}^{\prime}(\bar{x}) \frac{h_{x_{1}}^{4}}{4 !}+H_{2}^{\prime}(\bar{x}) \frac{h_{x_{0}}^{3}}{3 !}+H_{3}^{\prime}(\bar{x}) \frac{h_{x_{1}}^{3}}{3 !}\right]} u^{(4)}(\bar{x}) \\
& +\cdots
\end{aligned}
$$


Now, considering the Hermite shape functions over the reference element $\hat{I}=$ $[-1,+1]$ their derivatives in the optimal point are expressed as

$$
\begin{aligned}
& H_{0}^{\prime}(\bar{x})=\frac{1}{4}\left(-3+3 \bar{x}^{2}\right), \quad H_{1}^{\prime}(\bar{x})=\frac{1}{4}\left(3-3 \bar{x}^{2}\right), \\
& H_{2}^{\prime}(\bar{x})=\frac{1}{4}\left(-1-2 \bar{x}+3 \bar{x}^{2}\right), \quad H_{3}^{\prime}(\bar{x})=\frac{1}{4}\left(-1+2 \bar{x}+3 \bar{x}^{2}\right) .
\end{aligned}
$$

It is a simple algebrism to prove that the identity $I_{1}$ in equation (36) is null since that Hermite shape functions satisfy $H_{0}(x)+H_{1}(x)=1$ that implies $H_{0}^{\prime}(x)+H_{1}^{\prime}(x)=0$ after deriving in $x$. Substituting the expressions for $h_{x_{0}}$ and $h_{x_{1}}$ the identity $I_{2}$ in (36) becomes

$$
I_{2}=-H_{0}^{\prime}(\bar{x})+H_{1}^{\prime}(\bar{x})+H_{2}^{\prime}(\bar{x})-H_{3}^{\prime}(\bar{x})-\bar{x}\left[H_{0}^{\prime}(\bar{x})+H_{1}^{\prime}(\bar{x})\right]
$$

Noting that the wronskian of the Hermite's shape functions is not zero then set $\left\{H_{i}\right\}_{i=0}^{3}$ is a basis therefore, combining with the completeness property one follows that $x=x_{0} H_{0}(x)+x_{1} H_{1}(x)+H_{2}(x)+H_{3}(x)$ and if $x_{0}=-1, x_{1}=+1$ then $H_{2}^{\prime}(\bar{x})+H_{3}^{\prime}(\bar{x})=1+H_{0}^{\prime}(\bar{x})-H_{1}^{\prime}(\bar{x})$ that implies $I_{2}=1$ in (36).

Following the arguments above let us prove that the coefficient $I_{3}$ below

$$
\begin{aligned}
I_{3}= & \frac{1}{2}\left[H_{0}^{\prime}(\bar{x})+H_{1}^{\prime}(\bar{x})+\bar{x}^{2}\left(H_{0}^{\prime}(\bar{x})+H_{1}^{\prime}(\bar{x})\right)\right] \\
& +\bar{x}\left[H_{0}^{\prime}(\bar{x})-H_{1}^{\prime}(\bar{x})-H_{2}^{\prime}(\bar{x})+H_{3}^{\prime}(\bar{x})\right]-H_{2}^{\prime}(\bar{x})+H_{3}^{\prime}(\bar{x}) .
\end{aligned}
$$

is identically null. To do this note initially that $H_{0}^{\prime}(x)+H_{1}^{\prime}(x)=0$ and hence $I_{3}$ is given by

$$
I_{3}=-\bar{x} \underbrace{-H_{2}^{\prime}(\bar{x})+H_{3}^{\prime}(\bar{x})}_{\bar{x}}=0 .
$$

Starting from the analogous arguments and hypothesis it is possible to demonstrate the nulling of the coefficient $I_{4}$ in (36) expressed as

$$
\begin{aligned}
I_{4}= & -\frac{1}{2} \bar{x}\left[H_{0}^{\prime}(\bar{x})+H_{1}^{\prime}(\bar{x})\right]-\frac{1}{6} \bar{x}^{3}\left[H_{0}^{\prime}(\bar{x})+H_{1}^{\prime}(\bar{x})\right] \\
& -\frac{1}{6}\left[H_{0}^{\prime}(\bar{x})-H_{1}^{\prime}(\bar{x})\right]+-\frac{1}{2} \bar{x}^{2}\left[H_{0}^{\prime}(\bar{x})-H_{1}^{\prime}(\bar{x})\right] \\
& +\frac{1}{2}\left[H_{2}^{\prime}(\bar{x})+H_{3}^{\prime}(\bar{x})\right]+\bar{x}\left[H_{2}^{\prime}(\bar{x})-H_{3}^{\prime}(\bar{x})\right] \\
& +\frac{1}{2} \bar{x}^{2}\left[H_{2}^{\prime}(\bar{x})+H_{3}^{\prime}(\bar{x})\right] .
\end{aligned}
$$


By using the Hermitian basis properties in equation (42), one obtains

$$
I_{4}=\frac{1}{3} H_{0}^{\prime}(\bar{x})-\frac{1}{3} H_{1}^{\prime}(\bar{x})+\bar{x}\left[H_{2}^{\prime}(\bar{x})-H_{3}^{\prime}(\bar{x})\right]+\frac{1}{2} \bar{x}^{2}+\frac{1}{2}
$$

that implies $I_{4}=0$.

Surprisingly, the coefficient $I_{5}$ as defined in equation (36) is not identically null and it becomes after simplification

$$
I_{5}=\frac{1}{6}\left(x-x^{3}\right) .
$$

Assuming that $u \in C^{5}(\hat{I})$ then an $O\left(h^{5}\right)$ convergence is obtained at the points where $I_{5}(x)=0$, thus, the optimal points are $x=0, \pm 1$. Obviously, these points are exactly the nodal points where not only the nodal values $u_{0}$ and $u_{1}$ are coincident but also the derivative values $u_{0}^{\prime}$ and $u_{1}^{\prime}$. As expected, the centroid is an optimal point, exhibiting an $O\left(h^{k+2}\right)$ convergence, therefore, it is an ultracovergent point if Hermite's finite elements are used since $k=3$ in this particular case. Hence, Hermite's basis shows higher accuracy when compared with the Lagrange's finite element for which the centroid is only superconvergent while the former is ultraconvergent. It makes sense then that the superconvergence phenomenon depends on the basis selected.

\section{Barlow points for one dimensional Lagrange's elements}

The Barlow's Method [2] for computing the optimal points usually assumes that the exact polynomial displacement field is one order higher than that of the finite element field. The arbitrariness of this assumption is necessary in order to derive the coordinates of the optimal points in the sense of Barlow since the exact field is unknown for the real world problems.

Starting from the finite element displacement field written as $\overline{\mathbf{u}}=\mathbf{N} \overline{\mathbf{u}}_{\mathbf{n}}$ which in a polynomial form is expressed as

$$
\overline{\mathbf{u}}=\mathbf{p}(\mathbf{x})^{\mathbf{t}} \cdot \mathbf{a}
$$

where $\mathbf{N}$ is the shape function matrix, $\mathbf{p}(\mathbf{x})^{\mathbf{t}}$ is a matrix of monomial terms, and $\mathbf{a}$ is a vector of generalized parameters, after substituting the nodal co-ordinates 
of the element for $\mathbf{x}$ in equation (45), the nodal displacement, $\overline{\mathbf{u}}_{n}$, can be expressed as

$$
\overline{\mathbf{u}}_{n}=\mathbf{P a}
$$

where $\mathbf{P}$ is a matrix defined as $\mathbf{P}=\left[\mathbf{p}\left(\mathbf{x}_{1}\right), \ldots, \mathbf{p}\left(\mathbf{x}_{\mathbf{n}}\right)\right]^{\mathbf{t}}$ where $n$ is the number of nodes in the finite element, $\mathbf{x}_{\mathbf{i}}$ are the nodal point co-ordinate vectors. If the element geometry is such that the inverse of $\mathbf{P}$ exists, then, the equation (46) is rewritten as

$$
\mathbf{a}=\mathbf{P}^{-1} \overline{\mathbf{u}}_{\mathbf{n}}
$$

From equations (45) and (47) follows that

$$
\mathbf{N}=\mathbf{p}(\mathbf{x})^{\mathbf{t}} \mathbf{P}^{-1}
$$

Now let the exact displacement field be expressed as

$$
\mathbf{u}=\mathbf{p}(\mathbf{x})^{\mathbf{t}} \mathbf{b}+\mathbf{q}(\mathbf{x})^{\mathbf{t}} \mathbf{c}=\mathbf{r}(\mathbf{x})^{\mathbf{t}} \mathbf{d}
$$

where $\mathbf{r}(\mathbf{x})^{\mathbf{t}}=\left[\mathbf{p}(\mathbf{x})^{\mathbf{t}} \mathbf{q}(\mathbf{x})^{\mathbf{t}}\right]$ and $\mathbf{d}=[\mathbf{b} \mathbf{c}]^{\mathbf{t}}, \mathbf{q}(\mathbf{x})^{\mathbf{t}}$ is a matrix of monomial terms, and $\mathbf{c}$, the vector of arbitrary constants. After substituting the nodal coordinates $\mathbf{x}$ in equation (49), one obtains

$$
\mathbf{u}_{\mathbf{n}}=\mathbf{P b}+\mathbf{Q c}
$$

From equations (45) and (49), the finite element strain and exact strain field are expressed as

$$
\begin{aligned}
& \bar{\epsilon}_{n}=(\mathbf{L N}) \overline{\mathbf{u}}_{\mathbf{n}} \\
& \epsilon=\mathbf{L r}(\mathbf{x})^{\mathbf{t}} \mathbf{d}
\end{aligned}
$$

where $\mathbf{L}$ is a differential operator matrix. Using equation (48) and (50), equation (52) simplifies to

$$
\epsilon=\mathbf{L}\left(\mathbf{N} \mathbf{u}_{\mathbf{n}}-\mathbf{N Q c}+\mathbf{q}(\mathbf{x})^{\mathbf{t}} \mathbf{c}\right)
$$

The error in the stress, $\mathbf{e}_{\sigma}$, with the Barlow's hypothesis that $\overline{\mathbf{u}}_{n}=\mathbf{u}_{\mathbf{n}}$, is calculated by

$$
\left.\mathbf{e}_{\sigma}=\mathbf{D}(\bar{\epsilon}-\epsilon)=\mathbf{D L}\left(\mathbf{N Q}-\mathbf{q}(\mathbf{x})^{\mathbf{t}}\right) \mathbf{c}\right)
$$


Since $\mathbf{c}$ is an arbitrary constant, the Barlow Points are given by the zeros of the nonlinear vector equation

$$
\mathrm{DL}\left(\mathbf{N Q}-\mathbf{q}(\mathbf{x})^{\mathbf{t}}\right)=\mathbf{0}
$$

\subsection{Linear elements}

Using the linear lagrangian element, Equations (45), (46), (47) and (48) yield to

$$
\begin{aligned}
& \bar{u}=N \bar{u}_{n} \Leftrightarrow u_{h}=\left[\begin{array}{ll}
L_{1} & L_{2}
\end{array}\right] \cdot\left[\begin{array}{l}
u\left(x_{1}\right) \\
u\left(x_{2}\right)
\end{array}\right] \\
& \bar{u}=p(x)^{t} \cdot a \Leftrightarrow u_{h}=\left[\begin{array}{ll}
1 & x
\end{array}\right] \cdot\left[\begin{array}{l}
a_{1} \\
a_{2}
\end{array}\right] \\
& \begin{array}{l}
u\left(x_{1}\right)=a_{1}+a_{2} x_{1} \\
u\left(x_{2}\right)=a_{1}+a_{2} x_{2}
\end{array} \Leftrightarrow \underbrace{\left[\begin{array}{l}
u\left(x_{1}\right) \\
u\left(x_{2}\right)
\end{array}\right]}_{\bar{u}_{n}}=\underbrace{\left[\begin{array}{ll}
1 & x_{1} \\
1 & x_{2}
\end{array}\right]}_{P} \underbrace{\left[\begin{array}{l}
a_{1} \\
a_{2}
\end{array}\right]}_{a} .
\end{aligned}
$$

Obviously, $x_{1} \neq x_{2}$, then $\mathbf{P}^{-1}$ exists. Assuming that the exact field is one order higher than the linear finite element interpolant, then equation (49) gives

$$
\begin{aligned}
& u=\left[\begin{array}{ll}
1 & x
\end{array}\right] \cdot\left[\begin{array}{l}
b_{1} \\
b_{2}
\end{array}\right]+\left[x^{2}\right] \cdot\left[\begin{array}{l}
c_{1}
\end{array}\right] \\
& \begin{array}{l}
u\left(x_{1}\right)=b_{1}+b_{2} x_{1}+c_{1} x_{1}^{2} \\
u\left(x_{2}\right)=b_{1}+b_{2} x_{2}+c_{1} x_{2}^{2}
\end{array} \Leftrightarrow \underbrace{\left[\begin{array}{l}
u\left(x_{1}\right) \\
u\left(x_{2}\right)
\end{array}\right]}_{\bar{u}_{n}} \\
& =\underbrace{\left[\begin{array}{ll}
1 & x_{1} \\
1 & x_{2}
\end{array}\right]}_{P} \cdot \underbrace{\left[\begin{array}{l}
b_{1} \\
b_{2}
\end{array}\right]}_{b}+\underbrace{\left[\begin{array}{l}
x_{1}^{2} \\
x_{2}^{2}
\end{array}\right]}_{Q} \cdot \underbrace{\left[\begin{array}{l}
c_{1}
\end{array}\right]}_{c} \cdot \\
& u=L_{1}(x)\left[u\left(x_{1}\right)-c_{1} x_{1}^{2}\right]+L_{2}(x)\left[u\left(x_{2}\right)-c_{1} x_{2}^{2}\right]+x^{2} c_{1} .
\end{aligned}
$$


Deriving equation (61) and the linear finite element interpolant and substituting in equation of the error (54), one obtains

$$
\mathbf{e}_{\sigma}=\mathbf{c}_{1}\left(\mathbf{x}_{1}+\mathbf{x}_{2}-\mathbf{2 x}\right) .
$$

Thus, the Barlow point for a two-noded lagrangian element coincides with the centroid of the element.

\subsection{Quadratic elements}

Following the same procedure one has

$$
\begin{gathered}
\bar{u}=N \bar{u}_{n} \Leftrightarrow u_{h}=\left[\begin{array}{lll}
L_{1} & L_{2} & L_{3}
\end{array}\right] \cdot\left[\begin{array}{l}
u\left(x_{1}\right) \\
u\left(x_{2}\right) \\
u\left(x_{3}\right)
\end{array}\right] \\
\bar{u}=p(x)^{t} \cdot a \Leftrightarrow u_{h}=\left[\begin{array}{lll}
1 & x & x^{2}
\end{array}\right] \cdot\left[\begin{array}{l}
a_{1} \\
a_{2} \\
a_{3}
\end{array}\right] \\
\begin{array}{l}
u\left(x_{1}\right)=a_{1}+a_{2} x_{1}+a_{3} x_{1}^{2} \\
u\left(x_{2}\right)=a_{1}+a_{2} x_{2}+a_{3} x_{2}^{2} \\
u\left(x_{3}\right)=a_{1}+a_{2} x_{3}+a_{3} x_{3}^{2}
\end{array} \Leftrightarrow \underbrace{\left[\begin{array}{l}
u\left(x_{1}\right) \\
u\left(x_{2}\right) \\
u\left(x_{3}\right)
\end{array}\right]}_{\bar{u}_{n}}=\underbrace{\left[\begin{array}{lll}
1 & x_{1} & x_{1}^{2} \\
1 & x_{2} & x_{2}^{2} \\
1 & x_{3} & x_{3}^{2}
\end{array}\right]}_{P} \underbrace{\left[\begin{array}{l}
a_{1} \\
a_{2} \\
a_{3}
\end{array}\right]}_{a} .
\end{gathered}
$$

Putting in the form of equation (49) one obtains

$$
\begin{aligned}
& u=p(x)^{t} b+q(x)^{t} c=\left[\begin{array}{lll}
1 & x & x^{2}
\end{array}\right] \cdot\left[\begin{array}{l}
b_{1} \\
b_{2} \\
b_{3}
\end{array}\right]+\left[x^{3}\right] \cdot\left[c_{1}\right] . \\
& \begin{array}{l}
u\left(x_{1}\right)=b_{1}+b_{2} x_{1}+b_{3} x_{1}^{2}+c_{1} x_{1}^{3} \\
u\left(x_{2}\right)=b_{1}+b_{2} x_{2}+b_{3} x_{2}^{2}+c_{1} x_{2}^{3} \\
u\left(x_{3}\right)=b_{1}+b_{2} x_{3}+b_{3} x_{3}^{2}+c_{1} x_{3}^{3}
\end{array} \Leftrightarrow \underbrace{\left[\begin{array}{l}
u\left(x_{1}\right) \\
u\left(x_{2}\right) \\
u\left(x_{3}\right)
\end{array}\right]}_{\overline{u_{n}}} \\
& =\underbrace{\left[\begin{array}{lll}
1 & x_{1} & x_{1}^{2} \\
1 & x_{2} & x_{2}^{2} \\
1 & x_{3} & x_{3}^{2}
\end{array}\right]}_{P} \cdot \underbrace{\left[\begin{array}{l}
b_{1} \\
b_{2} \\
b_{3}
\end{array}\right]}_{b}+\underbrace{\left[\begin{array}{c}
x_{1}^{3} \\
x_{2}^{3} \\
x_{3}^{3}
\end{array}\right]}_{Q} \cdot \underbrace{\left[\begin{array}{l}
c_{1} \\
{[}
\end{array}\right.}_{c} .
\end{aligned}
$$


Hence, by deriving the exact displacement field

$$
\begin{aligned}
u= & L_{1}(x)\left[u\left(x_{1}\right)-c_{1} x_{1}^{3}\right]+L_{2}(x)\left[u\left(x_{2}\right)-c_{1} x_{2}^{3}\right] \\
& +L_{3}(x)\left[u\left(x_{3}\right)-c_{1} x_{3}^{3}\right]+x^{3} c_{1} .
\end{aligned}
$$

and substituting into the stress error $e_{\sigma}$ given by the equation (54), one obtains

$$
e_{\sigma}=c_{1}\left[L_{1}^{\prime}(x) x_{1}^{3}+L_{2}^{\prime}(x) x_{2}^{3}+L_{3}^{\prime}(x) x_{3}^{3}-3 x^{2}\right] \text {. }
$$

\subsection{Cubic elements}

In case of cubic lagrangian finite element from equations (45), (46), (47) and (48) follows that

$$
\begin{aligned}
& \bar{u}=N \bar{u}_{n} \Leftrightarrow u_{h}=\left[\begin{array}{llll}
L_{1} & L_{2} & L_{3} & L_{4}
\end{array}\right] \cdot\left[\begin{array}{l}
u\left(x_{1}\right) \\
u\left(x_{2}\right) \\
u\left(x_{3}\right) \\
u\left(x_{4}\right)
\end{array}\right] \\
& \bar{u}=p(x)^{t} \cdot a \Leftrightarrow u_{h}=\left[\begin{array}{llll}
1 & x & x^{2} & x^{3}
\end{array}\right] \cdot\left[\begin{array}{c}
a_{1} \\
a_{2} \\
a_{3} \\
a_{4}
\end{array}\right] \\
& u_{h}\left(x_{1}\right)=u\left(x_{1}\right)=a_{1}+a_{2} x_{1}+a_{3} x_{1}^{2}+a_{4} x_{1}^{3} \\
& u_{h}\left(x_{2}\right)=u\left(x_{2}\right)=a_{1}+a_{2} x_{2}+a_{3} x_{2}^{2}+a_{4} x_{2}^{3} \\
& u_{h}\left(x_{3}\right)=u\left(x_{3}\right)=a_{1}+a_{2} x_{3}+a_{3} x_{3}^{2}+a_{4} x_{3}^{3} \\
& u_{h}\left(x_{4}\right)=u\left(x_{4}\right)=a_{1}+a_{2} x_{4}+a_{3} x_{4}^{2}+a_{4} x_{4}^{3} \\
& \Leftrightarrow \underbrace{\left[\begin{array}{l}
u\left(x_{1}\right) \\
u\left(x_{2}\right) \\
u\left(x_{3}\right) \\
u\left(x_{4}\right)
\end{array}\right]}_{\bar{u}_{n}}=\underbrace{\left[\begin{array}{llll}
1 & x_{1} & x_{1}^{2} & x_{1}^{3} \\
1 & x_{2} & x_{2}^{2} & x_{2}^{3} \\
1 & x_{3} & x_{3}^{2} & x_{3}^{3} \\
1 & x_{4} & x_{4}^{2} & x_{4}^{3}
\end{array}\right]}_{P} \underbrace{\left[\begin{array}{c}
a_{1} \\
a_{2} \\
a_{3} \\
a_{4}
\end{array}\right]}_{a} .
\end{aligned}
$$


Using the equation (49) follows that

$$
\begin{gathered}
u=p(x)^{t} b+q(x)^{t} c \\
=\left[\begin{array}{llll}
1 & x & x^{2} & x^{3}
\end{array}\right] \cdot\left[\begin{array}{l}
b_{1} \\
b_{2} \\
b_{3} \\
b_{4}
\end{array}\right]+\left[x^{4}\right] \cdot\left[\begin{array}{l}
c_{1}
\end{array}\right] . \\
u\left(x_{1}\right)=b_{1}+b_{2} x_{1}+b_{3} x_{1}^{2}+b_{4} x_{1}^{3}+c_{1} x_{1}^{4} \\
u\left(x_{2}\right)=b_{1}+b_{2} x_{2}+b_{3} x_{2}^{2}+b_{4} x_{2}^{3}+c_{1} x_{2}^{4} \\
u\left(x_{3}\right)=b_{1}+b_{2} x_{3}+b_{3} x_{3}^{2}+b_{4} x_{3}^{3}+c_{1} x_{3}^{4} \\
\left.u\left(x_{4}\right)=b_{1}+b_{2} x_{4}+b_{3} x_{4}^{2}+b_{4} x_{4}^{3}+c_{1} x_{4}^{4} \begin{array}{c}
u\left(x_{1}\right) \\
u\left(x_{2}\right) \\
u\left(x_{3}\right) \\
u\left(x_{4}\right)
\end{array}\right] \\
=\underbrace{\left[\begin{array}{llll}
1 & x_{1} & x_{1}^{2} & x_{1}^{3} \\
1 & x_{2} & x_{2}^{2} & x_{2}^{3} \\
1 & x_{3} & x_{3}^{2} & x_{3}^{3} \\
1 & x_{4} & x_{4}^{2} & x_{4}^{3}
\end{array}\right]}_{P} \cdot \underbrace{\left[\begin{array}{c}
b_{1} \\
b_{2} \\
b_{3} \\
b_{4}
\end{array}\right]}_{b_{b}}+\underbrace{\left[\begin{array}{c}
x_{1}^{4} \\
x_{2}^{4} \\
x_{3}^{4} \\
x_{4}^{4}
\end{array}\right]}_{Q} \cdot \underbrace{\left[\begin{array}{l}
c \\
c_{1}
\end{array}\right]}_{c} .
\end{gathered}
$$

Deriving the exact displacement field given below

$$
\begin{aligned}
u= & L_{1}(x)\left[u\left(x_{1}\right)-c_{1} x_{1}^{4}\right]+L_{2}(x)\left[u\left(x_{2}\right)-c_{1} x_{2}^{4}\right] \\
& +L_{3}(x)\left[u\left(x_{3}\right)-c_{1} x_{3}^{4}\right]+L_{4}(x)\left[u\left(x_{4}\right)-c_{1} x_{4}^{4}\right]+x^{4} c_{1} .
\end{aligned}
$$

and substituting into the stress error expression given by (54), then

$$
e_{\sigma}=c_{1}\left[L_{1}^{\prime}(x) x_{1}^{4}+L_{2}^{\prime}(x) x_{2}^{4}+L_{3}^{\prime}(x) x_{3}^{4}+L_{4}^{\prime}(x) x_{4}^{4}-4 x^{3}\right] .
$$

\subsection{Fourth order elements}

Similarly, for fourth order lagrangian finite element from equations (45), (46), (47) and (48) follows that

$$
\bar{u}=N \bar{u}_{n} \Leftrightarrow u_{h}=\left[\begin{array}{lllll}
L_{1} & L_{2} & L_{3} & L_{4} & L_{5}
\end{array}\right] \cdot\left[\begin{array}{c}
u\left(x_{1}\right) \\
u\left(x_{2}\right) \\
u\left(x_{3}\right) \\
u\left(x_{4}\right) \\
u\left(x_{5}\right)
\end{array}\right]
$$




$$
\begin{aligned}
& \bar{u}=p(x)^{t} \cdot a \Leftrightarrow u_{h}=\left[\begin{array}{lllll}
1 & x & x^{2} & x^{3} & x^{4}
\end{array}\right] \cdot\left[\begin{array}{l}
a_{1} \\
a_{2} \\
a_{3} \\
a_{4} \\
a_{5}
\end{array}\right] \\
& u_{h}\left(x_{1}\right)=u\left(x_{1}\right)=a_{1}+a_{2} x_{1}+a_{3} x_{1}^{2}+a_{4} x_{1}^{3}+a_{5} x_{1}^{4} \\
& u_{h}\left(x_{2}\right)=u\left(x_{2}\right)=a_{1}+a_{2} x_{2}+a_{3} x_{2}^{2}+a_{4} x_{2}^{3}+a_{5} x_{2}^{4} \\
& u_{h}\left(x_{3}\right)=u\left(x_{3}\right)=a_{1}+a_{2} x_{3}+a_{3} x_{3}^{2}+a_{4} x_{3}^{3}+a_{5} x_{3}^{4} \\
& u_{h}\left(x_{4}\right)=u\left(x_{4}\right)=a_{1}+a_{2} x_{4}+a_{3} x_{4}^{2}+a_{4} x_{4}^{3}+a_{5} x_{4}^{4} \\
& u_{h}\left(x_{5}\right)=u\left(x_{5}\right)=a_{1}+a_{2} x_{5}+a_{3} x_{5}^{2}+a_{4} x_{5}^{3}+a_{5} x_{5}^{4}
\end{aligned}
$$

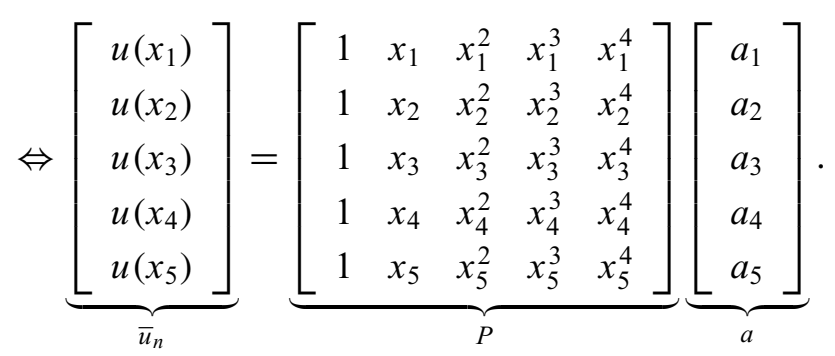

Again, by deriving the exact displacement field given below

$$
\begin{aligned}
u= & L_{1}(x)\left[u\left(x_{1}\right)-c_{1} x_{1}^{5}\right]+L_{2}(x)\left[u\left(x_{2}\right)-c_{1} x_{2}^{5}\right]+L_{3}(x)\left[u\left(x_{3}\right)-c_{1} x_{3}^{5}\right] \\
& +L_{4}(x)\left[u\left(x_{4}\right)-c_{1} x_{4}^{5}\right]+L_{5}(x)\left[u\left(x_{5}\right)-c_{1} x_{5}^{5}\right]+x^{5} c_{1} .
\end{aligned}
$$

and substituting into the stress error expression given by (54), then

$$
\mathbf{e}_{\sigma}=\mathbf{c}_{1}\left[\mathbf{L}_{1}^{\prime}(\mathbf{x}) \mathbf{x}_{1}^{5}+\mathbf{L}_{2}^{\prime}(\mathbf{x}) \mathbf{x}_{2}^{5}+\mathbf{L}_{3}^{\prime}(\mathbf{x}) \mathbf{x}_{3}^{5}+\mathbf{L}_{4}^{\prime}(\mathbf{x}) \mathbf{x}_{4}^{5}+\mathbf{L}_{5}^{\prime}(\mathbf{x}) \mathbf{x}_{5}^{5}-\mathbf{5} \mathbf{x}^{4}\right] .
$$

\subsection{Fifth order elements}

$$
\bar{u}=N \bar{u}_{n} \Leftrightarrow u_{h}=\left[\begin{array}{llllll}
L_{1} & L_{2} & L_{3} & L_{4} & L_{5} & L_{6}
\end{array}\right] \cdot\left[\begin{array}{c}
u\left(x_{1}\right) \\
u\left(x_{2}\right) \\
u\left(x_{3}\right) \\
u\left(x_{4}\right) \\
u\left(x_{5}\right) \\
u\left(x_{6}\right)
\end{array}\right]
$$




$$
\bar{u}=p(x)^{t} \cdot a \Leftrightarrow u_{h}=\left[\begin{array}{llllll}
1 & x & x^{2} & x^{3} & x^{4} & x^{5}
\end{array}\right] \cdot\left[\begin{array}{c}
a_{1} \\
a_{2} \\
a_{3} \\
a_{4} \\
a_{5} \\
a_{6}
\end{array}\right]
$$

Calculating $u_{h}$ in each node and expressing it in both basis, it turns out that $\bar{u}_{n}=P a \Rightarrow a=P^{-1} \cdot \bar{u}_{n}$. But the Vandermonde's matrix $P$

$$
P=\left[\begin{array}{llllll}
1 & x_{1} & x_{1}^{2} & x_{1}^{3} & x_{1}^{4} & x_{1}^{5} \\
1 & x_{2} & x_{2}^{2} & x_{2}^{3} & x_{2}^{4} & x_{2}^{5} \\
1 & x_{3} & x_{3}^{2} & x_{3}^{3} & x_{3}^{4} & x_{3}^{5} \\
1 & x_{4} & x_{4}^{2} & x_{4}^{3} & x_{4}^{4} & x_{4}^{5} \\
1 & x_{5} & x_{5}^{2} & x_{5}^{3} & x_{5}^{4} & x_{5}^{5} \\
1 & x_{6} & x_{6}^{2} & x_{6}^{3} & x_{6}^{4} & x_{6}^{5}
\end{array}\right]
$$

is invertible with equally spaced nodes. Hence, after substituting the exact field and the finite element interpolant field into the error equation in stress, one obtains

$$
\mathbf{e}_{\sigma}=\mathbf{c}_{1}\left[\mathbf{L}_{1}^{\prime}(\mathbf{x}) \mathbf{x}_{1}^{6}+\mathbf{L}_{2}^{\prime}(\mathbf{x}) \mathbf{x}_{2}^{6}+\mathbf{L}_{3}^{\prime}(\mathbf{x}) \mathbf{x}_{3}^{6}+\mathbf{L}_{4}^{\prime}(\mathbf{x}) \mathbf{x}_{4}^{6}+\mathbf{L}_{5}^{\prime}(\mathbf{x}) \mathbf{x}_{5}^{6}+\mathbf{L}_{6}^{\prime}(\mathbf{x}) \mathbf{x}_{6}^{6}-6 \mathbf{x}^{5}\right] .
$$

All analytical expressions for the error $\mathbf{e}_{\sigma}$ and the points where it is null are summarized in Table 6 and Table 7. By comparing Gauss points, Barlow points and Superconvergent points we can conclude that coordinates are different for each kind of optimal point, especially if higher order elements are used.

\section{Concluding remarks}

In this paper, Gaussian quadrature points, Superconvergent points in the sense of Taylor's Expansion and Barlow points are studied, proving the existence and summarizing the exact locations and convergence order as well for several spectral orders. As expected, the superconvergence property is not invariant under a changing in the node distribution inside the element, emphasizing the full importance of this open question related to non-uniform discretizations. The phenomenon of ultraconvergence, i.e. $O\left(h^{k+2}\right)$ convergence, is proved to be a 


\begin{tabular}{|c|l|}
\hline order $k$ & $\epsilon_{\sigma}(x)$ \\
\hline 1 & $2 c x$ \\
\hline 2 & $c\left(1-3 x^{2}\right)$ \\
\hline 3 & $c\left(\frac{20}{9} x-4 x^{3}\right)$ \\
\hline 4 & $\frac{c\left(-1+15 x^{2}-20 x^{4}\right)}{4}$ \\
\hline 5 & $\frac{2 c\left(-259 x+1750 x^{3}-1875 x^{5}\right)}{625}$ \\
\hline
\end{tabular}

Table 6 - Barlow's functions in 1D.

\begin{tabular}{|l|l|}
\hline $\mathrm{k}$ & Barlow's points \\
\hline 1 & 0 \\
\hline 2 & $\frac{ \pm \sqrt{3}}{3}$ \\
\hline 3 & $0, \pm \frac{\sqrt{5}}{3}$ \\
\hline 4 & $\pm \frac{\sqrt{3 \pm \sqrt{\frac{29}{5}}}}{2 \sqrt{2}}$ \\
\hline 5 & $0, \pm \frac{\sqrt{35 \pm 8 \sqrt{7}}}{5 \sqrt{3}}$ \\
\hline
\end{tabular}

Table 7 - Barlow's points.

numerical coincidence since the zeros of the superconvergence and ultraconvergence function are different in general if a Lagrange's Basis is used.

In case of Hermitian basis, the centroid shows an $O\left(h^{5}\right)$ convergence in the sense of Taylor Expansion, confirming that the center of the element is still the optimal position to calculate the derivative and more importantly a higher accuracy is also obtained from Hermitian finite element interpolants.

Two further points need to be discussed. The first is the invertibility of the isoparametric mapping which is related to the existence of superconvergent points if distorted and higher order elements are used. The natural question is now directed to the geometric transformation under which the reference element is mapped onto an arbitrary, general element. What assumptions should be imposed to the geometric mapping $x=F(\xi)$ in order to keep on the superconvergent point? In summary, what kind of geometric transformation can 
preserve the set of superconvergent points inside the element? It is a particularly important point since in finite element applications the mesh refinement leads to varying valence discretizations and several error indicators are based on gradient recovery and sampling superconvergent points [14]. The second point is that for higher spatial dimensions the complexity is seriously higher since the set of superconvergent points represents a superconvergence curve in 2D case or a superconvergence surface in 3D case. These topics will be considered in further discussion.

\section{REFERENCES}

[1] J. Barlow, Optimal stress locations in finite element models. Int. J. for Num. Meth. in Engng., 10 (1976), 243-251.

[2] K.M. Liew and S. Rajendran, New superconvergent points of the 8-node serendipity plane element for patch recovery. Int. J. for Num. Meth. in Engng., 54 (2002), 1103-1130.

[3] R.G. Mackinnon and G.F. Carey, Superconvergent derivatives: A Taylor Series Analysis. Int. J. for Num. Meth. in Engng., 28 (1989), 489-509.

[4] R.H. MacNeal, Finite Elements: Their Design and Performance. Marcel Dekker, New York (1994).

[5] D.S. Pinto Jr., Superconvergent Derivatives in Finite Element Method. PIBIC-CNPq/UFS, Technical Report (2001-2002) (in portuguese).

[6] D.S. Pinto Jr., On Higher Order Accuracy Points for First and Second Order Derivatives of Lagrangian Finite Element Interpolants. XXV CNMAC, Nova Friburgo, RJ (2002).

[7] D.S. Pinto Jr., Numerical and Analytical Studies of Superconvergence for First and Second Order Derivatives in Finite Element Interpolations. Procedings of $23^{\text {st }}$ Ibero-Latin American Congress on Computer Methods in Engineering, GiuliaNova, Italy (2002).

[8] D.S. Pinto Jr., The Superconvergence Points for Gradient and Hessian in the Quadrilateral Lagrangian Element. Procedings of $24^{\text {th }}$ Ibero-Latin American Congress on Computer Methods in Engineering, Ouro Preto/MG, Brazil (2003).

[9] D.S. Pinto Jr., Studies on Barlow Points, Gauss Points and Superconvergent Points in $1 D$ for Lagrange's Finite Elements. Procedings of $27^{\text {th }}$ Ibero-Latin American Congress on Computer Methods in Engineering, Campinas/SP, Brazil (2006).

[10] G. Prathap, Barlow Points and Gauss Points and the aliasing and best fit paradigms. Computers and Structures, 58 (1996), 321-325.

[11] G. Prathap, The Finite Element Method in Structural Mechanics. Kluwer Academic Publishers, Dordrecht, (1993). 
[12] G. Strang and GJ. Fix, An analysis of the finite element method. Prentice-Hall:Englewood Cliffs, NJ (1973).

[13] O.C. Zienkiewicz and J.Z. Zhu, The Superconvergent patch recovery and a posteriori error estimates. Part 1: The recovery technique. Int. J. for Num. Meth. in Engng., 33 (1992), 1331-1364.

[14] O.C. Zienkiewicz and J.Z. Zhu, The Superconvergent patch recovery and a posteriori error estimates. Part 2: Error Estimates and Adaptivity. Int. J. for Num. Meth. in Engng., 33 (1992), 1365-1382.

[15] M. Zlamal, Superconvergence and reduced integration in the finite element method. Math. Computations, 32 (1978), 663-685.

[16] X. Zhang, Why do Barlow points not coincide with the reduced Gauss quadrature points or higher-order finite elements. Commun. Numer. Meth. Engng., (in press). 